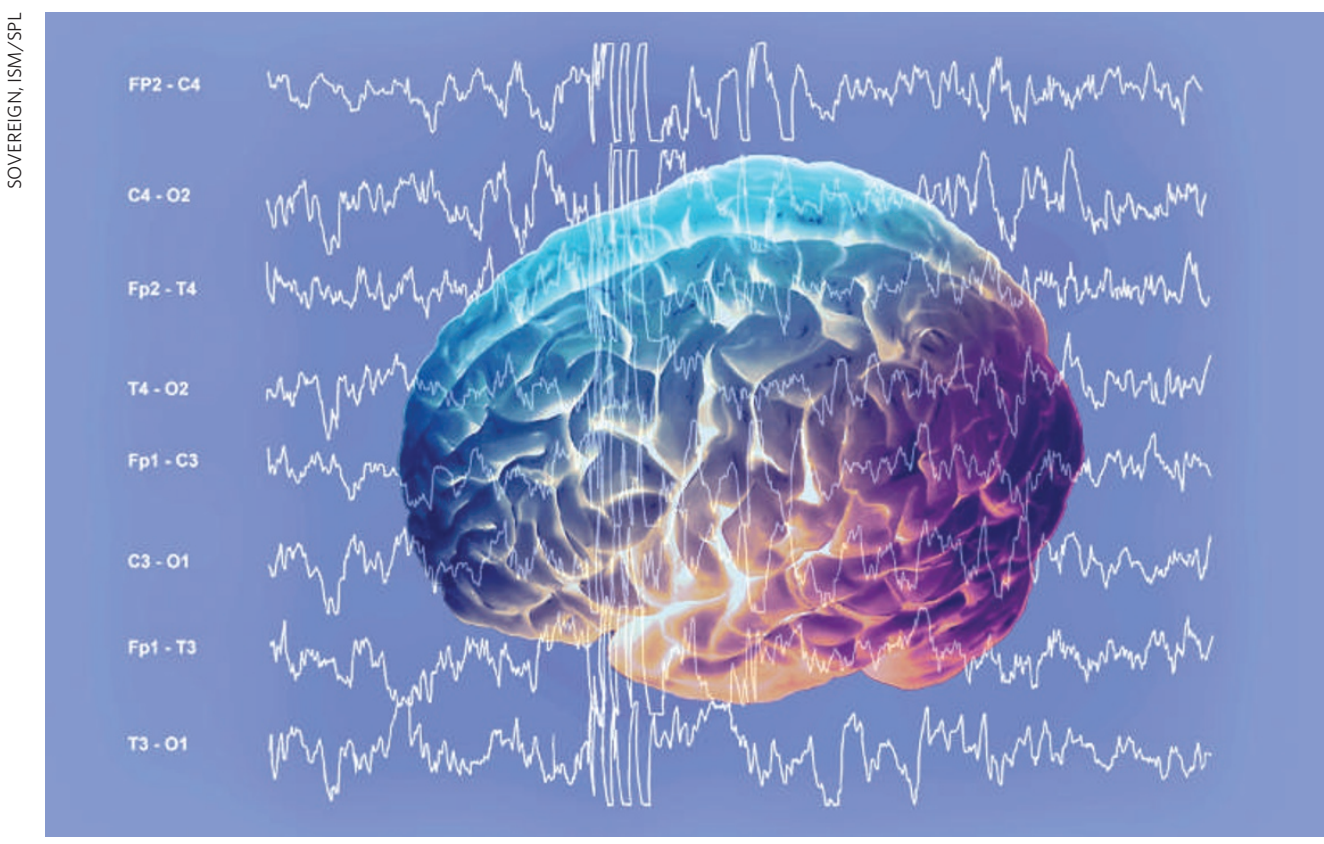

Brain waves are chaotic during an epileptic attack, as this electroencephalogram shows.

\title{
Fascinating rhythm
}

\section{Rhythms of the Brain}

by György Buzsáki

Oxford University Press: 2006. 464 pp.

$€ 42, \$ 69.95$

\section{Mayank Mehta}

The brain's primary task is probably to perceive environmental stimuli accurately and to generate appropriate responses quickly. Yet neurons not only respond to stimuli, but often do so in a rhythmic fashion. The strength of neural rhythms can predict a subject's performance on a task. Even when we sleep, neurons in most parts of the brain are active in a highly rhythmic fashion. By contrast, epileptic fits and Parkinson's disease are accompanied by an abnormal increase in certain brain rhythms.

What makes the brain rhythmic? What purpose do rhythms of the brain serve? These fascinating questions are being intensely investigated. In Rhythms of the Brain, György Buzsáki does a remarkable job of summarizing a vast body of literature on the topic.

This wonderful book seamlessly cycles between experimental results and theoretical concepts. It begins with an introduction to many topics related to brain rhythms, such as neuronal anatomy, physiology and the physics of self-organized systems. Here we learn about such things as scaling laws and complexity applied to simple neural circuits. Buzsáki describes a wide range of brain rhythms, ranging from very slow rhythms of the order of 1 cycle per second up to several hundred cycles per second. The frequency of rhythms changes as a function of development, ageing and disease. The frequency of oscillations often changes dramatically within a few seconds, as a function of the animal's behaviour. Subsequent chapters discuss various experimental methods used to measure neural activity, ranging from ion-channel measurements to multielectrode recordings and functional imaging, and their relative merits.

Buzsáki then moves on to describe possible functions of brain rhythms, such as resonance, synchronization of neural circuits, and improvement of signal-to-noise ratio by stochastic resonance. As with the rest of the book, these fascinating yet heavy topics are punctuated by entertaining stories, such as the origin of the word 'synchronicity', which refers to a key concept in systems neuroscience. According to Buzsáki, the physicist Wolfgang Pauli consulted the psychologist Carl Jung about the meaning of his dreams. After years of conversation, Pauli and Jung came up with the concept of synchronicity, which refers to the coincidence in time of multiple events that may not be causally unrelated.

The next few chapters summarize a vast amount of literature about the origin of neocortical oscillations and the joint effect of external stimuli and internally generated oscillations on perception. We learn that perception is largely governed by the brain's own internal, often involuntary, dynamics. Seemingly involuntary oscillations can dramatically enhance the perception of a stimulus. For example, neural activity is often modulated by a rhythm of $4-12 \mathrm{~Hz}$, called the theta rhythm. The presence of theta oscillations can generate a fivefold increase in a rabbit's ability to respond to a stimulus. Further, neocortical activity is often modulated by a rhythm of $40-80 \mathrm{~Hz}$, called the gamma rhythm. When there are strong gamma oscillations in certain parts of the neocortex, human subjects do better on learning and memory tasks.

The last few chapters are devoted to the functioning of a seahorse-shaped region called the hippocampus. This topic is the focus of Buzsáki's research, and he is one of the leading experts in this area. The hippocampus is essentially 'rhythm central', and hippocampal neurons probably show the widest range of oscillatory modulation. For example, these neurons mostly fire randomly when a rat sits around grooming itself. As soon as the rat starts to walk, the hippocampal neurons start to spike with a theta rhythm. The same theta rhythm appears when the rat is dreaming. When the rat stops walking and rests without dreaming, the theta rhythm is largely gone, and is replaced by occasional, short bursts of oscillations of about 200 cycles per second, called ripples. In addition to this range of rhythms, hippocampal neurons also fire selectively as a function of the rat's position in space. So, just by listening to the neural activity, one can gain information on where the rat is in space and whether it is resting or running. Hearing the rat hippocampus on a loudspeaker is an unforgettable experience. Buzsáki's book describes the amazing influence of oscillations on information encoding in the hippocampus and how this may be critical for learning facts and events. It ends with a discussion of some of the toughest problems in the field, such as what consciousness is, and how to irrefutably demonstrate the role of oscillations in brain function.

The book is clearly written and points the reader to the literature on a wide range of issues related to oscillations. Most chapters are accessible to a lay audience, and even practising neuroscientists would find much to learn here. The book is a 'must read' for anyone interested in understanding the functioning of large and complex brain circuits.

Mayank Mehta is in the Department of

Neuroscience, Brown University, Providence, Rhode Island 02912, USA.

http://neurophysics.brown.edu 